\title{
Analytic Approximate Solutions for MHD Boundary-Layer Viscoelastic Fluid Flow over Continuously Moving Stretching Surface by Homotopy Analysis Method with Two Auxiliary Parameters
}

\author{
M. M. Rashidi, ${ }^{1}$ E. Momoniat, ${ }^{2}$ and B. Rostami ${ }^{1}$ \\ ${ }^{1}$ Mechanical Engineering Department, Engineering Faculty of Bu-Ali Sina University, Hamedan, Iran \\ ${ }^{2}$ Centre for Differential Equations, Continuum Mechanics and Applications, \\ School of Computational and Applied Mathematics, University of the Witwatersrand, Johannesburg, \\ Private Bag 3, Wits 2050, South Africa
}

Correspondence should be addressed to M. M. Rashidi, mm_rashidi@yahoo.com

Received 3 August 2012; Accepted 15 September 2012

Academic Editor: Fazal M. Mahomed

Copyright (c) 2012 M. M. Rashidi et al. This is an open access article distributed under the Creative Commons Attribution License, which permits unrestricted use, distribution, and reproduction in any medium, provided the original work is properly cited.

In this study, a steady, incompressible, and laminar-free convective flow of a two-dimensional electrically conducting viscoelastic fluid over a moving stretching surface through a porous medium is considered. The boundary-layer equations are derived by considering Boussinesq and boundary-layer approximations. The nonlinear ordinary differential equations for the momentum and energy equations are obtained and solved analytically by using homotopy analysis method (HAM) with two auxiliary parameters for two classes of visco-elastic fluid (Walters' liquid B and second-grade fluid). It is clear that by the use of second auxiliary parameter, the straight line region in $\hbar$-curve increases and the convergence accelerates. This research is performed by considering two different boundary conditions: (a) prescribed surface temperature (PST) and (b) prescribed heat flux (PHF). The effect of involved parameters on velocity and temperature is investigated.

\section{Introduction}

The analysis of the flow field in a boundary layer near a stretching sheet is an important part in fluid dynamics and heat transfer occurring in a number of engineering processes such as polymer processing, metallurgy, extrusion of plastic sheets, and crystal growth $[1,2]$.

Incompressible MHD visco-elastic (Rivlin-Ericksen) fluid flow with small particles between two infinite moving parallel plates was analyzed via Laplace transform technique 
by Ghosh et al. [3]. Datti et al. [4] carried out a study on MHD visco-elastic fluid flow over a nonisothermal stretching sheet with presence of internal heat generation/absorption and radiation. The fourth-order Runge-Kutta method was applied to investigate the effects of suction/blowing on steady boundary-layer flow and heat transfer considering thermal radiation. The flow of non-Newtonian power-law fluids past a power-law stretched sheet with surface heat flux has been investigated by Chen [5]. A central-difference scheme was employed to solve governing equations and to discuss the effects of different physical parameters. Bég et al. [6] employed Keller-box implicit method to analyze Soret and Dufour effects on heat and mass transfer micropolar fluid flow over an isothermal sphere. MHD flow of an infinite vertical porous plate was investigated by Kamel [7] using Laplace transform techniques.

Computers have a significant effect on the developments of new methods. Today, researchers apply analytical methods in solving nonlinear problems and use many advantages of these methods like high convergence, and so forth. One of the most known and reliable techniques is homotopy analysis method. Homotopy analysis method (HAM) was employed by Liao, as the first one to offer a general analytic method for nonlinear problems $[8,9]$. Heat transfer of a magnetohydrodynamic Sisko fluid through a porous medium was studied by Khan and Farooq [10] and they compared a Sisko fluid to a Newtonian fluid. Rashidi and Pour [11] employed HAM for unsteady boundary-layer flow and heat transfer on a stretching sheet. Hayat et al. [12] investigated Soret and Dufour's effect on mixed convection of a visco-elastic fluid flow over a vertical stretching surface via HAM. Abbas et al. [13] studied mixed convection boundary-layer flow of a Maxwell fluid over a vertical stretching surface by HAM. Khan and Shahzad $[14,15]$ studied boundary-layer flow of a nonNewtonian (Sisko) fluid over a radially stretching sheet and a wedge via HAM respectively, considering involved parameters. Khan et al. [16] also considered the flow of a Sisko fluid in an annular pipe and solved both analytically (HAM) and numerically (the finite difference method). Partial slip, thermal diffusion, and diffusion thermo on MHD convective flow over a rotating disk with viscous dissipation and ohmic heating was studied by Rashidi et al. [17] via HAM. They clearly stated that increase in magnetic parameter leads to decrease in the radial skin friction and increase in slip coefficient leads to an increase in the heat transfer coefficient. HAM has been extensively used to solve nonlinear problems in mechanics and fluid dynamics [18-24].

To accelerate solution convergence and to improve the method, we use HAM with two auxiliary parameters. The second auxiliary parameter increases the straight line of $\hbar$-curve and the rate of convergence. Aliakbar et al. [25] surveyed the effects of involved parameters in MHD flow of Maxwellian fluids in presence of thermal radiation via HAM with two auxiliary parameters.

\section{Flow Analysis}

We consider a steady-state two-dimensional boundary-layer flow of an electrically conducting visco-elastic incompressible laminar-free convective fluid over a moving stretching surface in a porous medium. Two opposite and equal forces along $x$-axis are applied, keeping the origin fixed so the sheet is stretched [1]. The stretching velocity is assumed to be $u_{\omega}(x)=b x$. A uniform magnetic field along $y$-axis is imposed. Assuming magnetic Reynolds number very small, we neglect the induced magnetic field in comparison to the applied magnetic field. Viscous dissipation is small. With the Boussinesq and the boundary-layer 
approximations and considering the above assumptions, the boundary-layer equations are [1]:

$$
\begin{gathered}
\frac{\partial u}{\partial x}+\frac{\partial v}{\partial y}=0 \\
u \frac{\partial u}{\partial x}+v \frac{\partial u}{\partial y}=-k_{0}\left\{u \frac{\partial^{3} u}{\partial x \partial y^{2}}+v \frac{\partial^{3} u}{\partial y^{3}}+\frac{\partial u}{\partial x} \frac{\partial^{2} u}{\partial y^{2}}-\frac{\partial u}{\partial y} \frac{\partial^{2} u}{\partial x \partial y}\right\} \\
+v \frac{\partial^{2} u}{\partial y^{2}}-\frac{\sigma B_{0}^{2} u}{\rho}-\frac{v}{k^{\prime}} u+g \beta\left(T-T_{\infty}\right),
\end{gathered}
$$

where $u$ and $v$ are velocity components in the directions of $x$ and $y$ along and perpendicular to the surface, respectively. The boundary conditions are

$$
\begin{gathered}
u=u_{\omega}(x), \quad v=0, \quad \text { at } y=0, \\
u \longrightarrow 0, \quad u_{y} \longrightarrow 0, \quad \text { as } y \longrightarrow \infty .
\end{gathered}
$$

By introducing stream function $\psi$, that $u=\partial \psi / \partial y, v=-\partial \psi / \partial x$, the continuity equation is satisfied. The momentum and energy equations can be transformed to the ordinary differential equations by using the following introduced similarity solutions:

$$
\eta=\sqrt{\frac{u_{\omega}(x)}{v x}} y, \quad \psi=\sqrt{v x u_{\omega}(x)} f(\eta)
$$

$\eta$ is the nondimensional distance of boundary-layer and $f(\eta)$ is the dimensionless stream function. Substitution (2.3) into the boundary-layer equation, a similarity non-linear ordinary differential equation is obtained:

$$
\begin{aligned}
f_{\eta}^{2}(\eta)-f(\eta) f_{\eta \eta}(\eta)= & f_{\eta \eta \eta}(\eta)-k_{1}\left\{2 f_{\eta}(\eta) f_{\eta \eta \eta}(\eta)-f(\eta) f_{\eta \eta \eta \eta}(\eta)-f_{\eta \eta}^{2}(\eta)\right\} \\
& -\operatorname{Mn} f_{\eta}(\eta)-k_{2} f_{\eta}(\eta)+\operatorname{Gr} \theta(\eta)
\end{aligned}
$$

where subscript $\eta$ in equations and superscript ' in figures denote the derivative in respect to $k_{1}=k_{0} b / v$ is the visco-elastic parameter, $k_{2}=v / b k^{\prime}$ is the permeability parameter, $\mathrm{Mn}=$ $\sigma B_{0}^{2} / b \rho$ is magnetic field parameter, and $\mathrm{Gr}=g \beta A / b^{2} l$ is Grashof number. The boundary conditions are as follow:

$$
\begin{gathered}
f_{\eta}(\eta)=1, \quad f(\eta)=0, \quad \text { at } \eta=0, \\
f_{\eta}(\eta) \longrightarrow 0, \quad f_{\eta \eta}(\eta) \longrightarrow 0, \quad \text { as } \eta \longrightarrow \infty .
\end{gathered}
$$


If we want to apply reduction of order to (2.4) we have

$$
\begin{gathered}
f_{\eta}(\eta)=z(f), \\
f_{\eta \eta}(\eta)=z \frac{d z}{d f}, \\
f_{\eta \eta \eta}(\eta)=z \frac{d}{d f}\left(z \frac{d z}{d f}\right), \\
f_{\eta \eta \eta \eta}(\eta)=z \frac{d}{d f}\left(z \frac{d}{d f}\left(z \frac{d z}{d f}\right)\right),
\end{gathered}
$$

reducing (2.4) to

$$
\begin{aligned}
z^{2}-f z \frac{d z}{d f}= & z \frac{d}{d f}\left(z \frac{d z}{d f}\right)-k_{1}\left\{2 z^{2} \frac{d}{d f}\left(z \frac{d z}{d f}\right)-f z \frac{d}{d f}\left(z \frac{d}{d f}\left(z \frac{d z}{d f}\right)\right)-z^{2}\left(\frac{d z}{d f}\right)^{2}\right\} \\
& -\operatorname{Mn} z-k_{2} z+\operatorname{Gr} \theta(\eta) .
\end{aligned}
$$

This reduction is possible because (2.4) is autonomous and hence admits the symmetry generator $X=\partial / \partial \eta$. The related boundary conditions are as follows:

$$
\begin{gathered}
z(0)=1, \\
z \frac{d z}{d f} \longrightarrow 0, \quad \text { as } \eta \longrightarrow \infty, \\
z \longrightarrow 0, \quad \text { as } \eta \longrightarrow \infty .
\end{gathered}
$$

But we do not have any value for $f$ when $\eta \rightarrow \infty$, that is, $f(\infty)$ is unknown: therefore, we are unable to apply a symmetry reduction to this boundary value problem. For analytical solution of this problem, we implement the HAM in the Section 3.

\section{Heat Transfer}

The energy equation with radiation and heat generation/absorption for flow in two dimensions is

$$
u \frac{\partial T}{\partial x}+v \frac{\partial T}{\partial y}=\frac{1}{\rho C_{p}} \frac{\partial}{\partial y}\left(K \frac{\partial T}{\partial y}\right)+\frac{Q}{\rho C_{p}}\left(T-T_{\infty}\right)-\frac{1}{\rho C_{p}} \frac{\partial q_{r}}{\partial y}
$$

the thermal conductivity $K$ changes linearly respect to the temperature in the form of in

$$
K=K_{\infty}(1+\varepsilon \theta(\eta))
$$


where

$$
\varepsilon=\frac{K_{\omega}-K_{\infty}}{K_{\infty}}
$$

By the use of Rosseland approximation the radiative heat flux is given by,

$$
q_{r}=-\frac{4 \sigma^{*}}{3 K^{*}} \frac{\partial T^{4}}{\partial y}
$$

$\sigma^{*}$ is the Stephan-Boltzman constant and $K^{*}$ is the mean absorption coefficient. Assume that $T^{4}$ is defined as a linear function of temperature. Using Taylor series,

$$
T^{4} \cong 4 T_{\infty}^{3} T-3 T_{\infty}^{4}
$$

Considering two different types of heating processes we have the following.

\subsection{Prescribed Surface Temperature (PST)}

The boundary conditions in this case are

$$
\begin{gathered}
T=T_{\omega}=T_{\infty}+A\left(\frac{x}{l}\right), \quad \text { at } y=0, \\
T \longrightarrow T_{\infty} \text { as } y \longrightarrow \infty .
\end{gathered}
$$

$A$ is constant and $l$ is the characteristic length.

Defining nondimensional temperature as

$$
\theta(\eta)=\frac{T-T_{\infty}}{T_{\omega}-T_{\infty}}
$$

We can obtain dimensionless energy equation as follows:

$$
(1+\varepsilon \theta(\eta)+\mathrm{Nr}) \theta_{\eta \eta}(\eta)+\operatorname{Pr} f(\eta) \theta_{\eta}(\eta)-\operatorname{Pr}\left(f_{\eta}(\eta)-\alpha\right) \theta(\eta)+\varepsilon \theta_{\eta}^{2}(\eta)=0
$$

Dimensionless boundary conditions are

$$
\begin{gathered}
\theta(\eta)=1, \quad \text { at } \eta=0, \\
\theta(\eta) \longrightarrow 0, \quad \text { as } \eta \longrightarrow \infty
\end{gathered}
$$

$\operatorname{Pr}=\mu C_{p} / K_{\infty}$ is the Prandtl number, $\alpha=Q / b \rho C_{p}$ is heat source/sink parameter, and $\mathrm{Nr}=$ $16 \sigma^{*} T_{\infty}^{3} / 3 K^{*} K_{\infty}$ is the thermal radiation parameter. 


\subsection{Prescribed Wall Heat Flux (PHF)}

The corresponding boundary conditions are

$$
\begin{gathered}
-K\left(\frac{\partial T}{\partial y}\right)_{\omega}=Q_{\omega}=D\left(\frac{x}{l}\right), \quad \text { at } y=0, \\
T \longrightarrow T_{\infty} \quad \text { as } y \longrightarrow \infty .
\end{gathered}
$$

$Q_{\omega}$ is the wall heat flux and $D$ is a constant.

$$
\left(\frac{\partial T}{\partial y}\right)_{\omega}=-\frac{D}{K}\left(\frac{x}{l}\right)
$$

Now we can obtain that

$$
\begin{gathered}
\left(T_{\omega}-T_{\infty}\right)=\frac{D}{K}\left(\frac{x}{l}\right) \sqrt{\frac{v}{b}}, \\
\left(T-T_{\infty}\right)=\frac{D}{K}\left(\frac{x}{l}\right) \sqrt{\frac{v}{b}} g(\eta) .
\end{gathered}
$$

Defining non-dimensional temperature as

$$
g(\eta)=\frac{T-T_{\infty}}{T_{\omega}-T_{\infty}}
$$

Dimensionless form of energy equation and boundary conditions are

$$
\begin{gathered}
(1+\varepsilon g(\eta)+\mathrm{Nr}) g_{\eta \eta}(\eta)+\operatorname{Pr} f(\eta) g_{\eta}(\eta)-\operatorname{Pr}\left(f_{\eta}(\eta)-\alpha\right) g(\eta)+\varepsilon g_{\eta}^{2}(\eta)=0, \\
g_{\eta}(\eta)=-1, \quad \text { at } \eta=0, \\
g(\eta) \longrightarrow 0, \quad \text { as } \eta \longrightarrow \infty .
\end{gathered}
$$

\section{HAM Solution}

We choose the initial approximations to satisfy the boundary conditions. Use of two auxiliary parameters increases the rate of convergence of the solution. The appropriate initial approximations are as follows:

$$
\begin{gathered}
f_{0}(\eta)=\frac{1-e^{-r \eta}}{r}, \\
\theta_{0}(\eta)=e^{-r \eta}, \\
g_{0}(\eta)=\frac{e^{-r \eta}}{r} .
\end{gathered}
$$


Journal of Applied Mathematics

The linear operators $\mathcal{L}_{f}(f), \mathcal{L}_{\theta}(\theta)$ and $\mathcal{L}_{g}(g)$, are defined as

$$
\begin{aligned}
& \mathcal{L}_{f}(f)=\frac{\partial^{4} f}{\partial \eta^{4}}+\gamma \frac{\partial^{3} f}{\partial \eta^{3}} \\
& \mathcal{L}_{\theta}(\theta)=\frac{\partial^{2} \theta}{\partial \eta^{2}}+\gamma \frac{\partial \theta}{\partial \eta^{\prime}} \\
& \mathcal{L}_{g}(g)=\frac{\partial^{2} g}{\partial \eta^{2}}+\gamma \frac{\partial g}{\partial \eta} .
\end{aligned}
$$

The following properties are satisfied with the above linear operators:

$$
\begin{gathered}
\mathcal{L}_{f}\left(c_{1}+c_{2} \eta+c_{3} \eta^{2}+c_{4} e^{-\gamma \eta}\right)=0, \\
\mathcal{L}_{\theta}\left(c_{5}+c_{6} e^{-\gamma \eta}\right)=0, \\
\mathcal{L}_{g}\left(c_{7}+c_{8} e^{-\gamma \eta}\right)=0 .
\end{gathered}
$$

$c_{1}-c_{8}$ are arbitrary constants. The nonlinear operators are

$$
\begin{aligned}
\mathcal{N}_{f}[\widehat{f}(\eta ; q), \widehat{\theta}(\eta ; q)] & \left(\frac{\partial \widehat{f}(\eta ; q)}{\partial \eta}\right)^{2}-\widehat{f}(\eta ; q) \frac{\partial^{2} \widehat{f}(\eta ; q)}{\partial \eta^{2}}-\frac{\partial^{3} \widehat{f}(\eta ; q)}{\partial \eta^{3}} \\
& +k_{1}\left\{2 \frac{\partial \widehat{f}(\eta ; q)}{\partial \eta} \frac{\partial^{3} \widehat{f}(\eta ; q)}{\partial \eta^{3}}-\widehat{f}(\eta ; q) \frac{\partial^{4} \widehat{f}(\eta ; q)}{\partial \eta^{4}}-\left(\frac{\partial^{2} \widehat{f}(\eta ; q)}{\partial \eta^{2}}\right)^{2}\right\} \quad \text { PST case } \\
& +\operatorname{Mn} \frac{\partial \widehat{f}(\eta ; q)}{\partial \eta}+k_{2} \frac{\partial \widehat{f}(\eta ; q)}{\partial \eta}-\operatorname{Gr} \widehat{\theta}(\eta ; q) \\
\mathcal{N}_{f}[\widehat{f}(\eta ; q), \widehat{g}(\eta ; q)] & \left(\frac{\partial \widehat{f}(\eta ; q)}{\partial \eta}\right)^{2}-\widehat{f}(\eta ; q) \frac{\partial^{2} \widehat{f}(\eta ; q)}{\partial \eta^{2}}-\frac{\partial^{3} \widehat{f}(\eta ; q)}{\partial \eta^{3}} \\
& +k_{1}\left\{2 \frac{\partial \widehat{f}(\eta ; q)}{\partial \eta} \frac{\partial^{3} \widehat{f}(\eta ; q)}{\partial \eta^{3}}-\widehat{f}(\eta ; q) \frac{\partial^{4} \widehat{f}(\eta ; q)}{\partial \eta^{4}}-\left(\frac{\partial^{2} \widehat{f}(\eta ; q)}{\partial \eta^{2}}\right)^{2}\right\} \quad \text { PHF case } \\
& +\operatorname{Mn} \frac{\partial \widehat{f}(\eta ; q)}{\partial \eta}+k_{2} \frac{\partial \widehat{f}(\eta ; q)}{\partial \eta}-\operatorname{Gr} \widehat{g}(\eta ; q),
\end{aligned}
$$




$$
\begin{aligned}
& \mathcal{N}_{\theta}[\widehat{f}(\eta ; q), \widehat{\theta}(\eta ; q)] \\
&=(1+\varepsilon \hat{\theta}(\eta ; q)+\operatorname{Nr}) \frac{\partial^{2} \widehat{\theta}(\eta ; q)}{\partial \eta^{2}}+\operatorname{Pr} \widehat{f}(\eta ; q) \frac{\partial \widehat{\theta}(\eta ; q)}{\partial \eta} \\
&-\operatorname{Pr}\left(\frac{\partial \widehat{f}(\eta ; q)}{\partial \eta}-\alpha\right) \hat{\theta}(\eta ; q)+\varepsilon\left(\frac{\partial \widehat{\theta}(\eta ; q)}{\partial \eta}\right)^{2} \\
& \mathcal{N}_{g}[\widehat{f}(\eta ; q), \hat{g}(\eta ; q)] \\
&=(1+\varepsilon \widehat{g}(\eta ; q)+\operatorname{Nr}) \frac{\partial^{2} \widehat{g}(\eta ; q)}{\partial \eta^{2}}+\operatorname{Pr} \widehat{f}(\eta ; q) \frac{\partial \widehat{g}(\eta ; q)}{\partial \eta} \\
& \quad-\operatorname{Pr}\left(\frac{\partial \widehat{f}(\eta ; q)}{\partial \eta}-\alpha\right) \widehat{g}(\eta ; q)+\varepsilon\left(\frac{\partial \widehat{g}(\eta ; q)}{\partial \eta}\right)^{2} .
\end{aligned}
$$

The zero-order deformation equations are defined as

$$
\begin{gathered}
(1-q) \mathcal{L}_{f}\left[\widehat{f}(\eta ; q)-f_{0}(\eta)\right]=q \hbar \mathscr{\ell}_{f}(\eta) \mathcal{N}_{f}[\widehat{f}(\eta ; q), \widehat{\theta}(\eta ; q)], \quad \text { PST case } \\
(1-q) \mathcal{L}_{f}\left[\widehat{f}(\eta ; q)-f_{0}(\eta)\right]=q \hbar \mathscr{\ell}_{f}(\eta) \mathcal{N}_{f}[\widehat{f}(\eta ; q), \widehat{g}(\eta ; q)], \quad \text { PHF case } \\
(1-q) \mathcal{L}_{\theta}\left[\widehat{\theta}(\eta ; q)-\theta_{0}(\eta)\right]=q \hbar \mathscr{\ell}_{\theta}(\eta) \mathcal{N}_{\theta}[\widehat{f}(\eta ; q), \widehat{\theta}(\eta ; q)], \\
(1-q) \mathcal{L}_{g}\left[\hat{g}(\eta ; q)-g_{0}(\eta)\right]=q \hbar \mathscr{\ell}_{g}(\eta) \mathcal{N}_{g}[\widehat{f}(\eta ; q), \hat{g}(\eta ; q)] .
\end{gathered}
$$

$\hbar$ is auxiliary nonzero parameter and $\mathscr{l}_{f}(\eta), \mathscr{l}_{\theta}(\eta), \mathscr{l}_{g}(\eta)$ are auxiliary functions, which we chose them as

$$
\begin{gathered}
\mathscr{\ell}_{f}(\eta)=e^{-\gamma \eta}, \\
\mathscr{\ell}_{\theta}(\eta)=e^{-\gamma \eta}, \\
\mathscr{H}_{g}(\eta)=e^{-\gamma \eta}, \\
f_{m}(\eta)=\left.\frac{1}{m !} \frac{\partial^{m} \widehat{f}(\eta ; q)}{\partial q^{m}}\right|_{q=0}, \\
\theta_{m}(\eta)=\left.\frac{1}{m !} \frac{\partial^{m} \hat{\theta}(\eta ; q)}{\partial q^{m}}\right|_{q=0}, \\
g_{m}(\eta)=\left.\frac{1}{m !} \frac{\partial^{m} \widehat{g}(\eta ; q)}{\partial q^{m}}\right|_{q=0} .
\end{gathered}
$$


Differentiating the zero-order deformation equations, $m$ times in respect to $q$, and dividing by $m$ ! in $q=0$, we have the $m$ th-order deformation equations

$$
\begin{aligned}
& \mathcal{L}_{f}\left[f_{m}(\eta)-x_{m} f_{m-1}(\eta)\right]=\hbar \mathscr{\ell}_{f}(\eta) R_{f, m}(\eta), \\
& \mathcal{L}_{\theta}\left[\theta_{m}(\eta)-x_{m} \theta_{m-1}(\eta)\right]=\hbar \mathscr{\ell}_{\theta}(\eta) R_{\theta, m}(\eta), \\
& \mathcal{L}_{g}\left[g_{m}(\eta)-x_{m} g_{m-1}(\eta)\right]=\hbar \mathscr{\ell}_{g}(\eta) R_{g, m}(\eta),
\end{aligned}
$$

where

$$
\begin{aligned}
& R_{f, m}(\eta) \\
& =\sum_{n=0}^{m-1}\left(\frac{\partial f_{n}(\eta)}{\partial \eta} \frac{\partial f_{m-1-n}(\eta)}{\partial \eta}-f_{n}(\eta) \frac{\partial^{2} f_{m-1-n}(\eta)}{\partial \eta^{2}}\right)-\frac{\partial^{3} f_{m-1}(\eta)}{\partial \eta^{3}}+\sum_{n=0}^{m-1} k_{1} \\
& \times\left(2 \frac{\partial f_{n}(\eta)}{\partial \eta} \frac{\partial^{3} f_{m-1-n}(\eta)}{\partial \eta^{3}}-f_{n}(\eta) \frac{\partial^{4} f_{m-1-n}(\eta)}{\partial \eta^{4}}-\frac{\partial^{2} f_{n}(\eta)}{\partial \eta^{2}} \frac{\partial^{2} f_{m-1-n}(\eta)}{\partial \eta^{2}}\right) \quad \text { PST case } \\
& +\operatorname{Mn} \frac{\partial f_{m-1}(\eta)}{\partial \eta}+k_{2} \frac{\partial f_{m-1}(\eta)}{\partial \eta}-\operatorname{Gr} \theta_{m-1}(\eta) \\
& R_{f, m}(\eta) \\
& =\sum_{n=0}^{m-1}\left(\frac{\partial f_{n}(\eta)}{\partial \eta} \frac{\partial f_{m-1-n}(\eta)}{\partial \eta}-f_{n}(\eta) \frac{\partial^{2} f_{m-1-n}(\eta)}{\partial \eta^{2}}\right)-\frac{\partial^{3} f_{m-1}(\eta)}{\partial \eta^{3}}+\sum_{n=0}^{m-1} k_{1} \\
& \times\left(2 \frac{\partial f_{n}(\eta)}{\partial \eta} \frac{\partial^{3} f_{m-1-n}(\eta)}{\partial \eta^{3}}-f_{n}(\eta) \frac{\partial^{4} f_{m-1-n}(\eta)}{\partial \eta^{4}}-\frac{\partial^{2} f_{n}(\eta)}{\partial \eta^{2}} \frac{\partial^{2} f_{m-1-n}(\eta)}{\partial \eta^{2}}\right) \quad \text { PHF case } \\
& +\operatorname{Mn} \frac{\partial f_{m-1}(\eta)}{\partial \eta}+k_{2} \frac{\partial f_{m-1}(\eta)}{\partial \eta}-\operatorname{Grg}_{m-1}(\eta) \\
& R_{\theta, m}(\eta) \\
& =(1+\mathrm{Nr}) \frac{\partial^{2} \theta_{m-1}(\eta)}{\partial \eta^{2}}+\varepsilon \sum_{n=0}^{m-1} \theta_{n}(\eta) \frac{\partial^{2} \theta_{m-1-n}(\eta)}{\partial \eta^{2}} \\
& +\operatorname{Pr}\left(\sum_{n=0}^{m-1} f_{n}(\eta) \frac{\partial \theta_{m-1-n}(\eta)}{\partial \eta}-\theta_{n}(\eta) \frac{\partial f_{m-1-n}(\eta)}{\partial \eta}\right)+(\alpha \cdot \operatorname{Pr}) \theta_{m-1}(\eta) \\
& +\varepsilon \sum_{n=0}^{m-1} \frac{\partial \theta_{n}(\eta)}{\partial \eta} \frac{\partial \theta_{m-1-n}(\eta)}{\partial \eta}
\end{aligned}
$$




$$
\begin{aligned}
R_{g, m}(\eta) & \\
= & (1+\mathrm{Nr}) \frac{\partial^{2} g_{m-1}(\eta)}{\partial \eta^{2}}+\varepsilon \sum_{n=0}^{m-1} g_{n}(\eta) \frac{\partial^{2} g_{m-1-n}(\eta)}{\partial \eta^{2}} \\
& +\operatorname{Pr}\left(\sum_{n=0}^{m-1} f_{n}(\eta) \frac{\partial g_{m-1-n}(\eta)}{\partial \eta}-g_{n}(\eta) \frac{\partial f_{m-1-n}(\eta)}{\partial \eta}\right)+(\alpha \cdot \operatorname{Pr}) g_{m-1}(\eta) \\
& +\varepsilon \sum_{n=0}^{m-1} \frac{\partial g_{n}(\eta)}{\partial \eta} \frac{\partial g_{m-1-n}(\eta)}{\partial \eta} .
\end{aligned}
$$

Finally, we obtain by Taylor series the following:

$$
\begin{gathered}
\widehat{f}(\eta ; q)=f_{0}(\eta)+\sum_{m=1}^{\infty} f_{m}(\eta) q^{m}, \\
\widehat{\theta}(\eta ; q)=\theta_{0}(\eta)+\sum_{m=1}^{\infty} \theta_{m}(\eta) q^{m}, \\
\widehat{g}(\eta ; q)=g_{0}(\eta)+\sum_{m=1}^{\infty} g_{m}(\eta) q^{m}, \\
x_{m}= \begin{cases}0 & m \leq 1, \\
1 & m>1 .\end{cases}
\end{gathered}
$$

The system of equations with boundary conditions is solved by software MATHEMATICA.

\section{Convergence of HAM}

The appropriate values of the parameters $\hbar$ and $\gamma$ have significant influence on the solution convergence [8]. The optimal values are selected from the valid region in straight line. Straight line in $\hbar$-curve can be extended by the use of the second auxiliary parameter $\gamma$.

In Figure $1, \hbar$-curve is figured and obtained via 20th-order of HAM solution. We should select the optimal values from the straight lines parallel to the horizontal axis to control the convergence. Figures 2, 3, 4, and 5 obviously prove the effect of choosing the appropriate second auxiliary parameter in increasing the straight valid region of $\hbar$-curves. The averaged residual errors are defined as (5.1) to acquire optimal values of auxiliary parameters

$$
\begin{aligned}
\operatorname{Res}_{f}= & \left(\frac{d f(\eta)}{d \eta}\right)^{2}-f(\eta) \frac{d^{2} f(\eta)}{d \eta^{2}}-\frac{d^{3} f(\eta)}{d \eta^{3}} \\
& +k_{1}\left\{2 \frac{d f(\eta)}{d \eta} \frac{d^{3} f(\eta)}{d \eta^{3}}-f(\eta) \frac{d^{4} f(\eta)}{d \eta^{4}}-\left(\frac{d^{2} f(\eta)}{d \eta^{2}}\right)^{2}\right\}
\end{aligned}
$$




$$
\begin{aligned}
& +\operatorname{Mn} \frac{d f(\eta)}{d \eta}+k_{2} \frac{d f(\eta)}{d \eta}-\operatorname{Gr} \theta(\eta), \quad \text { PST case } \\
\operatorname{Res}_{f}= & \left(\frac{d f(\eta)}{d \eta}\right)^{2}-f(\eta) \frac{d^{2} f(\eta)}{d \eta^{2}}-\frac{d^{3} f(\eta)}{d \eta^{3}} \\
& +k_{1}\left\{2 \frac{d f(\eta)}{d \eta} \frac{d^{3} f(\eta)}{d \eta^{3}}-f(\eta) \frac{d^{4} f(\eta)}{d \eta^{4}}-\left(\frac{d^{2} f(\eta)}{d \eta^{2}}\right)^{2}\right\} \\
& +\operatorname{Mn} \frac{d f(\eta)}{d \eta}+k_{2} \frac{d f(\eta)}{d \eta}-\operatorname{Gr} g(\eta), \operatorname{PHF} \text { case } \\
\operatorname{Res}_{\theta}= & (1+\varepsilon \theta(\eta)+\operatorname{Nr}) \frac{d^{2} \theta(\eta)}{d \eta^{2}}+\operatorname{Pr} f(\eta) \frac{d \theta(\eta)}{d \eta} \\
& -\operatorname{Pr}\left(\frac{d f(\eta)}{d \eta}-\alpha\right) \theta(\eta)+\varepsilon\left(\frac{d \theta(\eta)}{d \eta}\right)^{2} \\
\operatorname{Res}_{g}= & (1+\varepsilon g(\eta)+\operatorname{Nr}) \frac{d^{2} g(\eta)}{d \eta^{2}}+\operatorname{Pr} f(\eta) \frac{d g(\eta)}{d \eta} \\
& -\operatorname{Pr}\left(\frac{d f(\eta)}{d \eta}-\alpha\right) g(\eta)+\varepsilon\left(\frac{d g(\eta)}{d \eta}\right)^{2}
\end{aligned}
$$

To check the accuracy of the method, the residual errors of the equations are illustrated in Figures 6(a), and 6(b). As one can see, the residual error is reduced when we use the second auxiliary parameter and this justifies why we use the second auxiliary parameter. In Figure 6(a) the effect of considering $\gamma=0.92$ in PHF case and $\gamma=0.93$ in PST case is to decrease the order of residual errors more than $\gamma=1$ (without the second auxiliary parameter) in Figure 6(b) and this improves the accuracy of HAM method. In fact, the selection of second auxiliary parameter has a determinative effect on the solution and improves the convergence strength of HAM. The second auxiliary parameter not only extends the straight line region in $\hbar$-curve but also leads to a better choice of $\hbar$ and hence improves the accuracy of the solution.

\section{Results and Discussion}

In this paper, the MHD boundary layer problem for momentum and heat transfer with buoyancy force, thermal radiation and internal heat source/sink in visco-elastic fluid flow (Walters' liquid B and second-grade fluid) over a porous stretching sheet is investigated.

The effect of different parameters on velocity and temperature distributions is discussed by applying numerical values to the involved parameters. Graphical representation of results is very useful to demonstrate the effect of different parameters in solutions. Figures 7 and 8 indicate the influence of the visco-elastic parameter $k_{1}$, in both PST and PHF cases on velocity profile. Visco-elasticity introduces tensile stress so the boundary-layer contracts transversely and hence velocity decreases. As we expect in Figures 7 and 8, velocity 


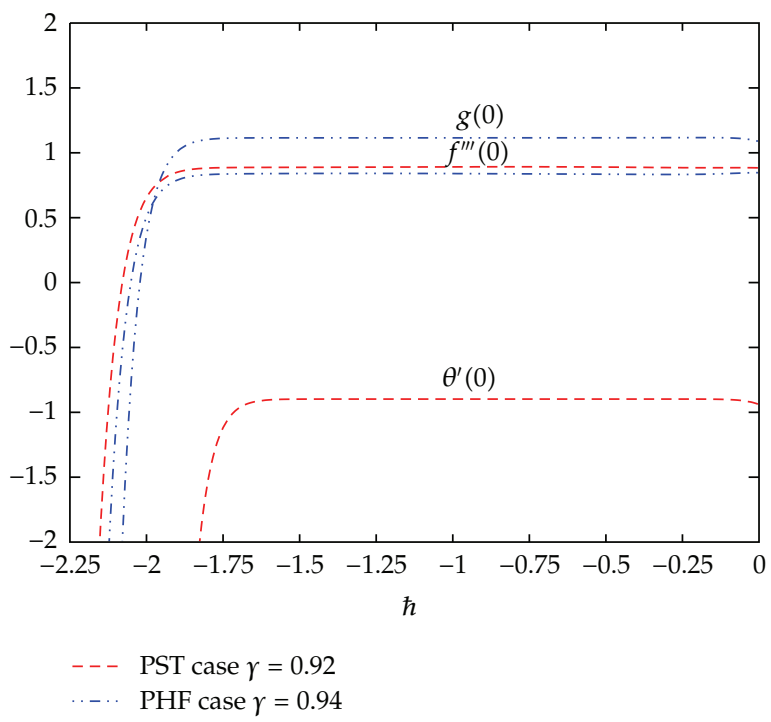

Figure 1: The $\hbar$-curves of $f^{\prime \prime \prime}(0), \theta^{\prime}(0)$ and $g(0)$ obtained by the 20th-order approximation of the HAM solution when $k_{1}=k_{2}=\mathrm{Nr}=\mathrm{Mn}=\varepsilon=0.1, \operatorname{Pr}=1, \alpha=0, \mathrm{Gr}=0.4$.

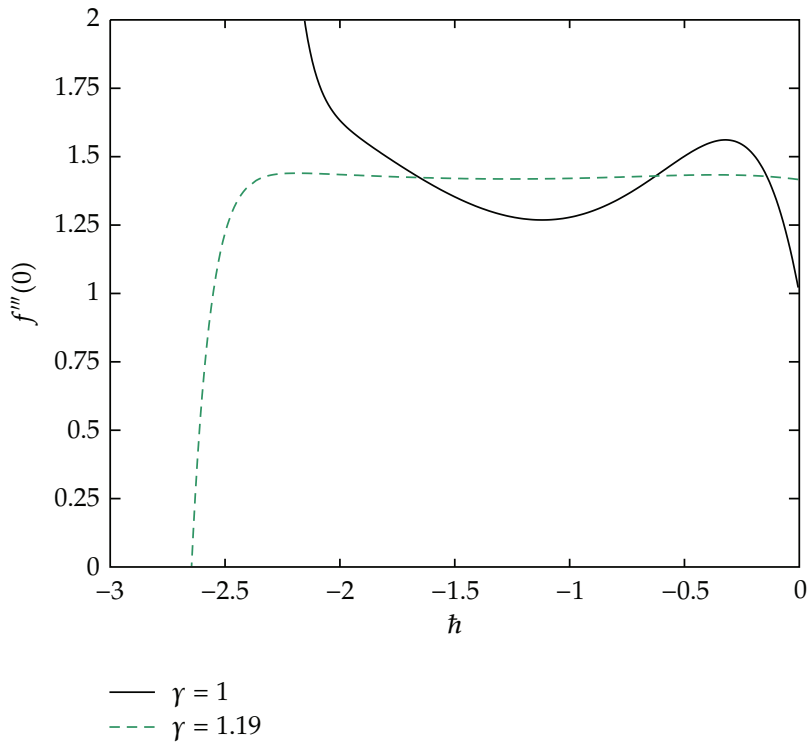

Figure 2: The effect of second auxiliary parameter on $\hbar$-curves of $f^{\prime \prime \prime}(0)$ when $\mathrm{Mn}=k_{2}=0.1, k_{1}=0.3$, $\mathrm{Gr}=0.2, \mathrm{Nr}=0.0, \mathrm{Pr}=1.0, \varepsilon=0.05, \alpha=-0.5$ in PST case.

decreases with increasing visco-elastic parameter. Considering both kinds of visco-elastic fluids (Walters' liquid B and second-grade fluid), we selected $k_{1}$, to vary from -0.5 to +0.5 .

The effect of magnetic parameter on flow is figured in Figure 9. Transverse magnetic field yields to create a drag like force named Lorentz force to resist the flow so magnetic field parameter slows down the flow and causes to decrease the horizontal velocity. We can see that in Figure 9 with increasing Mn, the velocity profiles decrease. The effect of permeability 


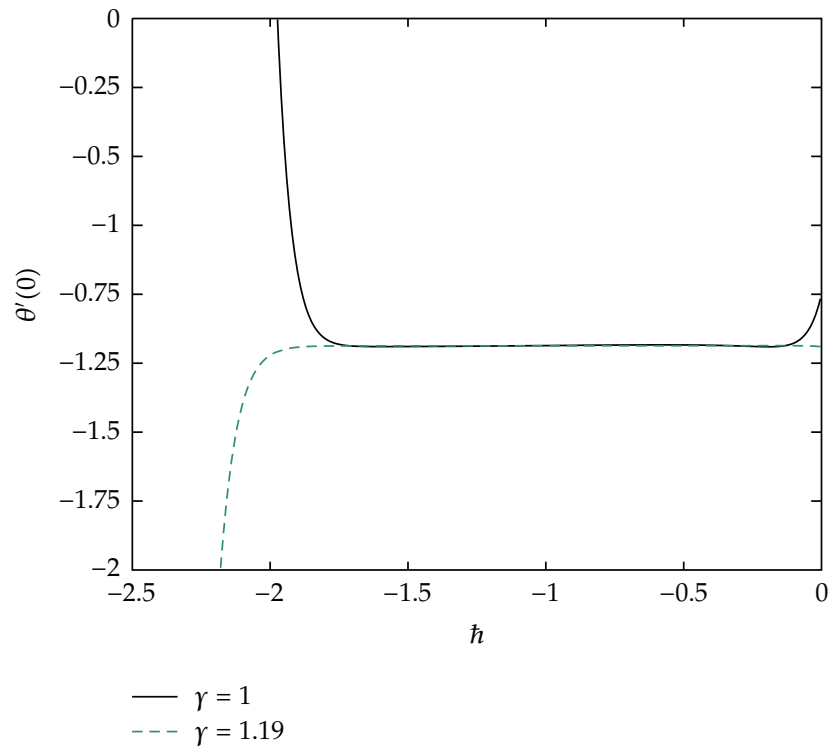

Figure 3: The effect of second auxiliary parameter on $\hbar$-curves of $\theta^{\prime}(0)$ when $\mathrm{Mn}=k_{2}=0.1, k_{1}=0.3$, $\mathrm{Gr}=0.2, \mathrm{Nr}=0.0, \mathrm{Pr}=1.0, \varepsilon=0.05, \alpha=-0.5$ in PST case.

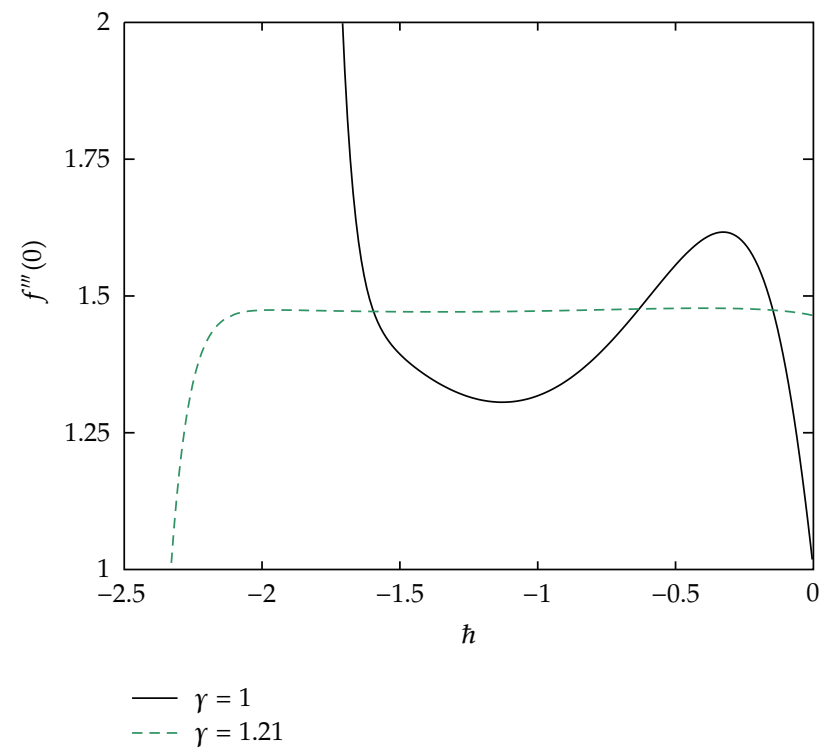

Figure 4: The effect of second auxiliary parameter on $\hbar$-curves of $f^{\prime \prime \prime}(0)$ when $\mathrm{Mn}=k_{2}=0.1, k_{1}=0.3$, $\mathrm{Gr}=0.2, \mathrm{Nr}=0.0, \mathrm{Pr}=1.0, \varepsilon=0.05, \alpha=-0.5$ in PHF case.

parameter is just like the effect of magnetic parameter. From Figure 10, one can obtain that with the increase of Grashof number velocity profiles increase. We should notice that $\mathrm{Gr}$ shows the coupling of the equations or the volumetric expansion capability of the fluid. Buoyancy force acts like a favorable pressure gradient and accelerates the fluid. With the increase of Prandtl number kinematic viscosity increases and velocity in both PST and PHF 


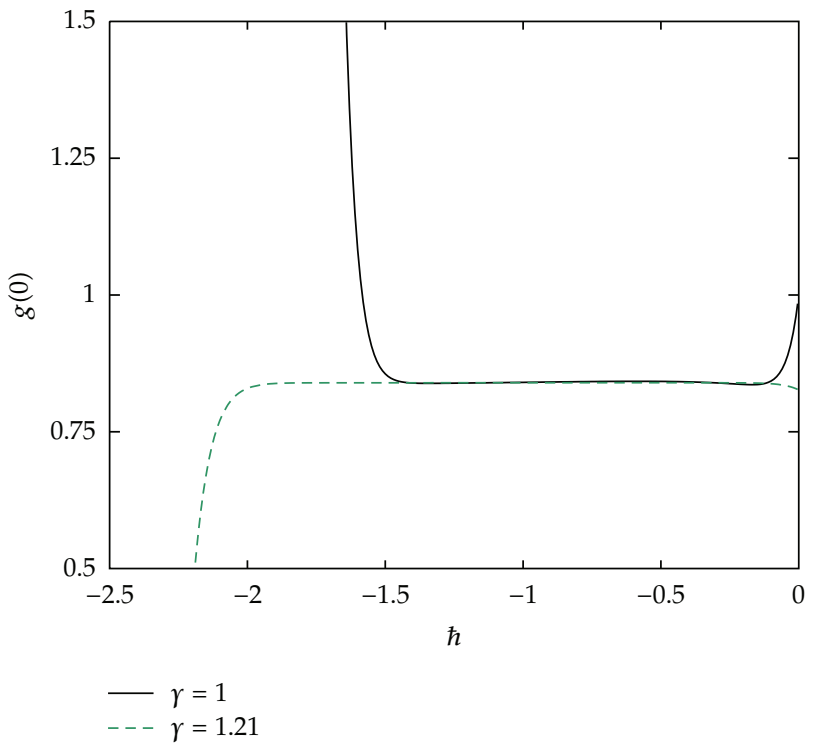

Figure 5: The effect of second auxiliary parameter on $\hbar$-curves of $g(0)$ when $\mathrm{Mn}=k_{2}=0.1, k_{1}=0.3$, $\mathrm{Gr}=0.2, \mathrm{Nr}=0.0, \mathrm{Pr}=1.0, \varepsilon=0.05, \alpha=-0.5$ in PHF case.

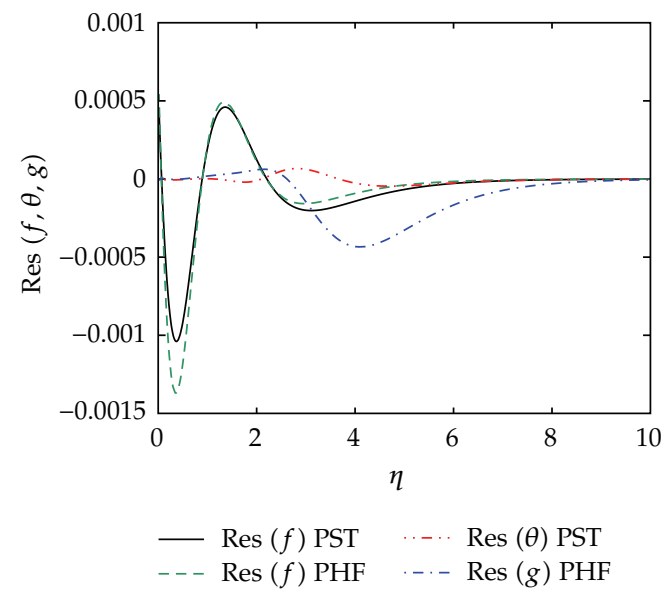

(a)

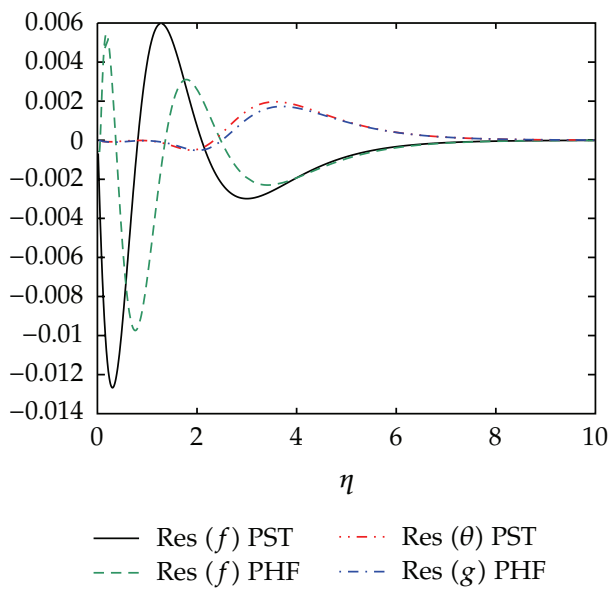

(b)

Figure 6: (a) The residual error when $k_{1}=-0.1, \mathrm{Mn}=0.2, k_{2}=0.0, \mathrm{Gr}=0.25, \mathrm{Pr}=1.0, \mathrm{Nr}=0.5, \varepsilon=0.15$, $\alpha=-0.3$, and $\gamma=0.93$ in PST case and $\gamma=0.92$ in PHF case. (b) The residual error when $k_{1}=-0.1$, $\mathrm{Mn}=0.2, k_{2}=0.0, \mathrm{Gr}=0.25, \mathrm{Pr}=1.0, \mathrm{Nr}=0.5, \varepsilon=0.15, \alpha=-0.3$, and $\gamma=1$ in both PST and PHF cases.

decreases. In Figure 11, it is shown that with the increase in Pr, the velocity profiles descends. The effect of thermal radiation parameter $\mathrm{Nr}$, on velocity profiles, is to increase the horizontal velocity that is shown in Figure 12.

The effect of heat source/sink parameter on velocity profile is to increase the velocity. It is very clear that with increasing $\alpha$ we can say that $b$ is decreasing and so the velocity should increase. 


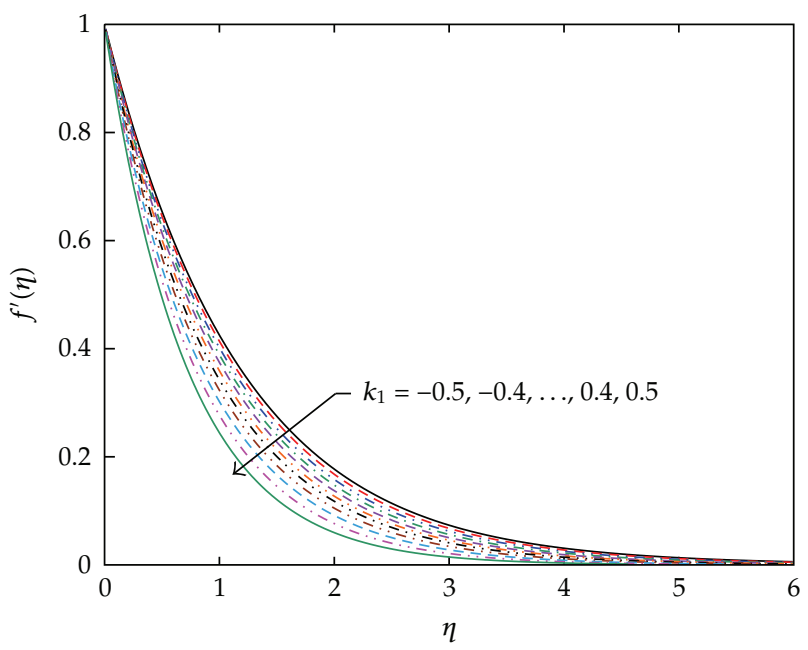

Figure 7: The velocity profile when $\mathrm{Mn}=0.15, k_{2}=0.05, \mathrm{Gr}=0.2, \mathrm{Pr}=1.0, \mathrm{Nr}=0.0, \varepsilon=0.05, \alpha=-0.5$. in PST case.

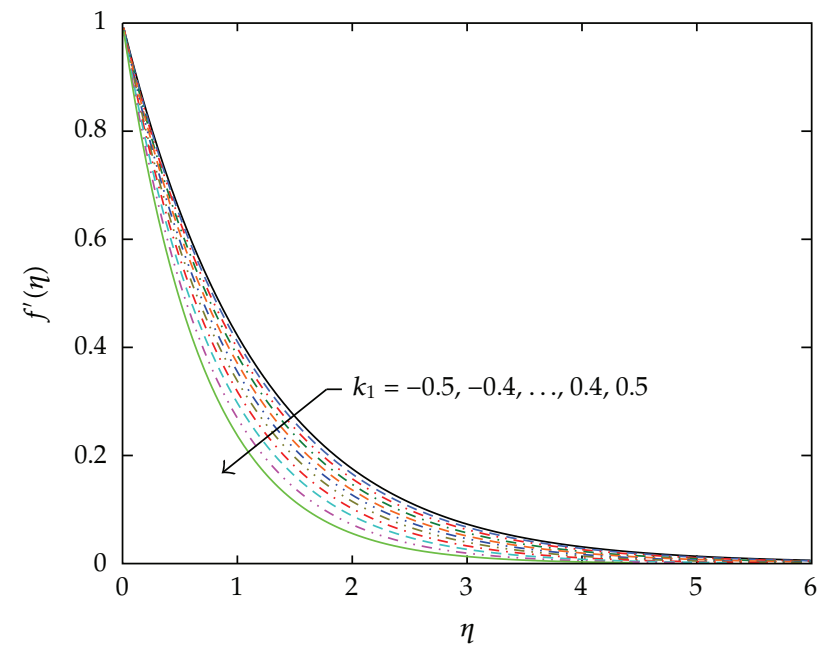

Figure 8: The velocity profile when $\mathrm{Mn}=0.15, k_{2}=0.05, \mathrm{Gr}=0.2, \mathrm{Pr}=1.0, \mathrm{Nr}=0.0, \varepsilon=0.05, \alpha=-0.5$ in PHF case.

With the increase of visco-elastic parameter as we expect, the temperature increases in both PST and PHF cases. When $(\alpha>0)$, that is, $T_{w}>T_{\infty}$ heat transfer occurs from sheet to fluid and temperature increases (we have energy generation). But when $(\alpha<0)$ heat transfer occurs from fluid to sheet (energy absorption) and temperature decreases. As we expect, the temperature in boundary layer in $\alpha>0$ is more than $\alpha<0$.

\section{Conclusion}

In this paper, we study the effect of the buoyancy force and thermal radiation in MHD boundary layer visco-elastic fluid flow over a continuously moving stretching surface in 


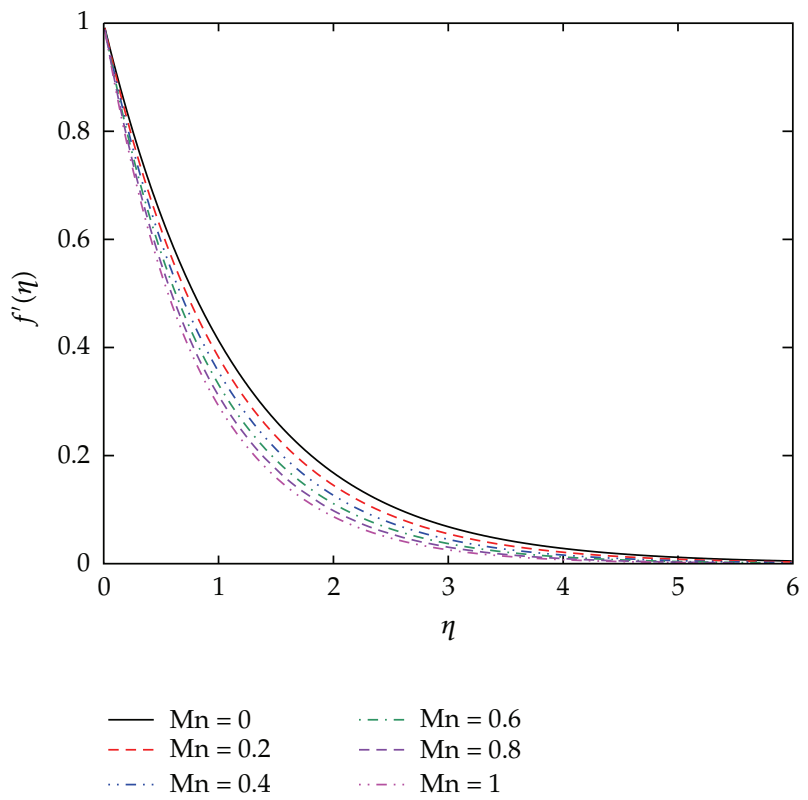

Figure 9: The velocity profile when $k_{1}=-0.2, k_{2}=0.1, \mathrm{Gr}=0.2, \mathrm{Pr}=1.0, \mathrm{Nr}=0.2, \varepsilon=0.1, \alpha=-0.25$ in PST case.

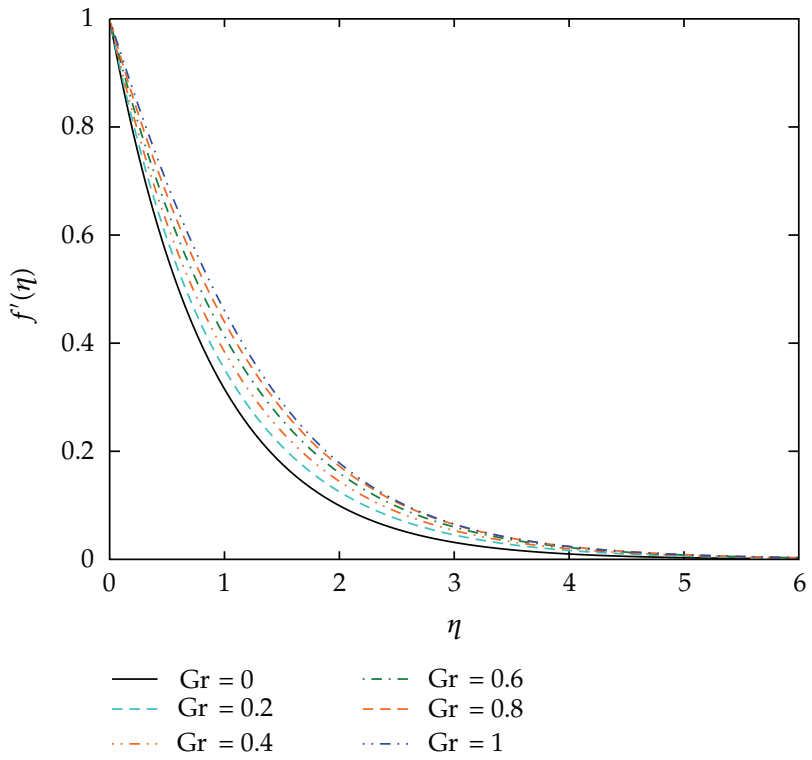

Figure 10: The velocity profile when $k_{1}=0.1, k_{2}=\mathrm{Nr}=0.0, \mathrm{Mn}=0.2, \mathrm{Pr}=1.0, \varepsilon=0.1, \alpha=-0.05$ in PST case.

a porous medium. The governing equations are formulated and the obtained equations transformed to ordinary differential equations and solved analytically using HAM for two classes of visco-elastic fluid (Walters' liquid B and second-grade fluid). The influence of the different parameters on the horizontal velocity and temperature profiles in two different boundary conditions (i) PST case and (ii) PHF case is illustrated and discussed. In general 


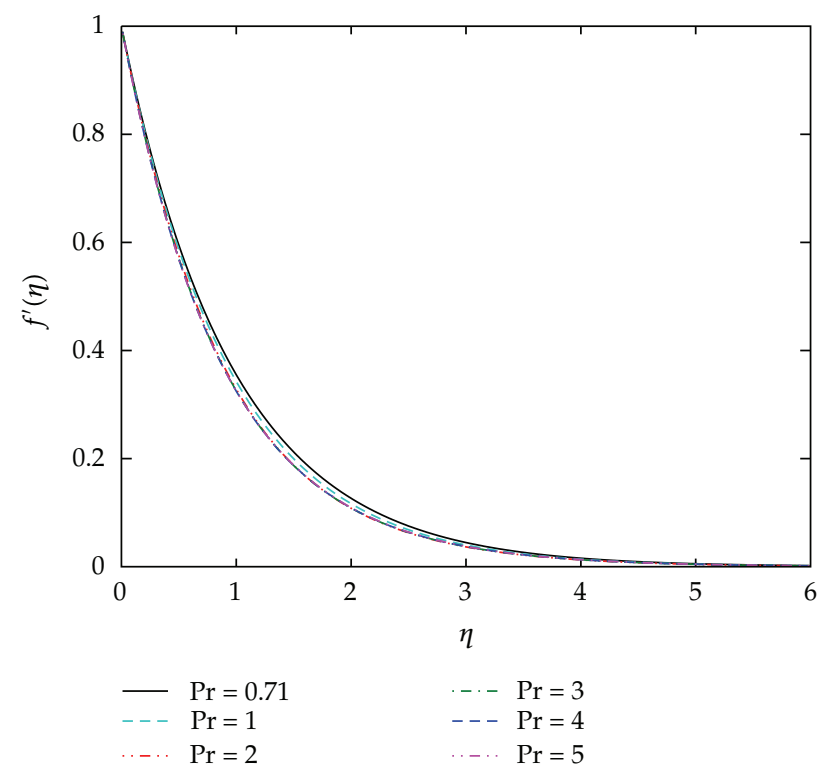

Figure 11: The velocity profile when $k_{1}=0.1, k_{2}=0.05, \mathrm{Mn}=0.1, \mathrm{Gr}=0.15, \mathrm{Nr}=0.0, \varepsilon=0.2, \alpha=-0.5$ in PHF case.

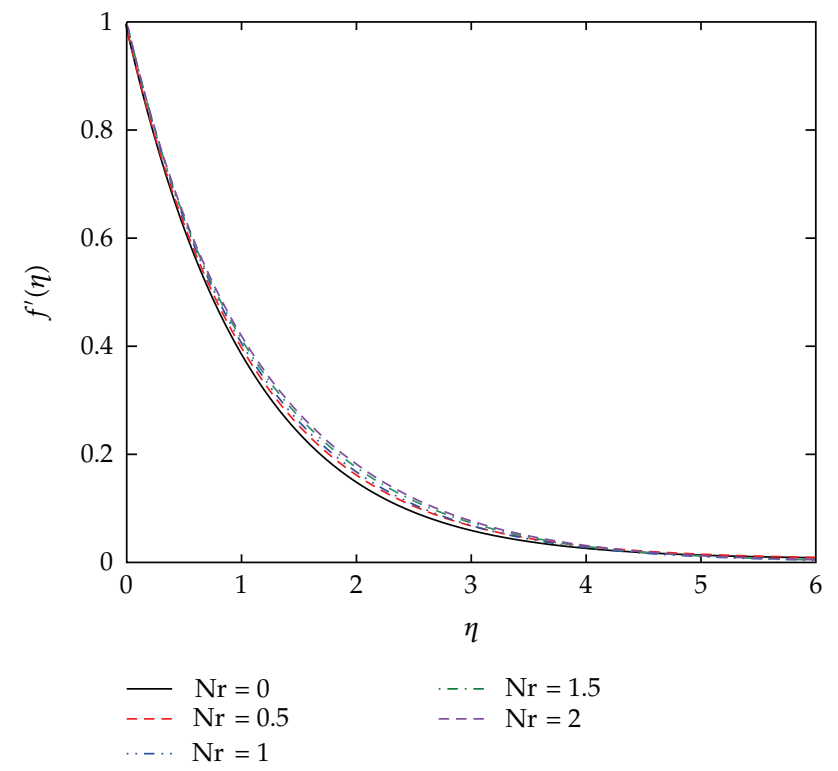

Figure 12: The velocity profile when $k_{1}=-0.1, k_{2}=0.2, \mathrm{Mn}=0.0, \mathrm{Gr}=0.25, \operatorname{Pr}=1.0, \varepsilon=0.15, \alpha=-0.3$ in PST case.

we note that the effect of visco-elastic parameter is to decrease the velocity and increase the temperature in boundary-layer. This mirrors the effect of magnetic field parameter. But with increasing the Prandtl number both velocity and temperature decrease. In PHF case, the wall temperature is greater than the PST case. 


\section{Acknowledgments}

M. M. Rashidi wishes to thank the Centre for Differential Equations, Continuum Mechanics and Applications, School of Computational and Applied Mathematics, University of the Witwatersrand, for the hospitality during his visit. E. Momoniat acknowledges support from the National Research Foundation of South Africa.

\section{References}

[1] S. Abel, K. V. Prasad, and A. Mahaboob, "Buoyancy force and thermal radiation effects in MHD boundary layer visco-elastic fluid flow over continuously moving stretching surface," International Journal of Thermal Sciences, vol. 44, no. 5, pp. 465-476, 2005.

[2] R. Tamizharasi and V. Kumaran, "Pressure in MHD/Brinkman flow past a stretching sheet," Communications in Nonlinear Science and Numerical Simulation, vol. 16, no. 12, pp. 4671-4681, 2011.

[3] N. C. Ghosh, B. C. Ghosh, and L. Debnath, "The hydromagnetic flow of a dusty visco-elastic fluid between two infinite parallel plates," Computers $\mathcal{E}$ Mathematics with Applications, vol. 39, no. 1-2, pp. 103-116, 2000.

[4] P. S. Datti, K. V. Prasad, M. S. Abel, and A. Joshi, “MHD visco-elastic fluid flow over a non-isothermal stretching sheet," International Journal of Engineering Science, vol. 42, no. 8-9, pp. 935-946, 2004.

[5] C. H. Chen, "Effects of magnetic field and suction/injection on convection heat transfer of nonNewtonian power-law fluids past a power-law stretched sheet with surface heat flux," International Journal of Thermal Sciences, vol. 47, no. 7, pp. 954-961, 2008.

[6] O. A. Bég, V. R. Prasad, B. Vasu, N. B. Reddy, Q. Li, and R. Bhargava, “Free convection heat and mass transfer from an isothermal sphere to a micropolar regime with Soret/Dufour effects," International Journal of Heat and Mass Transfer, vol. 54, no. 1-3, pp. 9-18, 2011.

[7] M. H. Kamel, "Unsteady MHD convection through porous medium with combined heat and mass transfer with heat source/sink," Energy Conversion and Management, vol. 42, no. 4, pp. 393-405, 2001.

[8] S. Liao, Beyond Perturbation: Introduction to the Homotopy Analysis Method, vol. 2, Chapman \& Hall, New York, NY, USA, 2004.

[9] S. Liao, "On the homotopy analysis method for nonlinear problems," Applied Mathematics and Computation, vol. 147, no. 2, pp. 499-513, 2004.

[10] M. Khan and J. Farooq, “On heat transfer analysis of a magneto-hydrodynamic sisko fluid through a porous medium," Journal of Porous Media, vol. 13, no. 3, pp. 287-294, 2010.

[11] M. M. Rashidi and S. A. M. Pour, "Analytic approximate solutions for unsteady boundary-layer flow and heat transfer due to a stretching sheet by homotopy analysis method," Lithuanian Association of Nonlinear Analysts, vol. 15, no. 1, pp. 83-95, 2010.

[12] T. Hayat, M. Mustafa, and I. Pop, "Heat and mass transfer for Soret and Dufour's effect on mixed convection boundary layer flow over a stretching vertical surface in a porous medium filled with a viscoelastic fluid," Communications in Nonlinear Science and Numerical Simulation, vol. 15, no. 5, pp. 1183-1196, 2010.

[13] Z. Abbas, Y. Wang, T. Hayat, and M. Oberlack, "Mixed convection in the stagnation-point flow of a Maxwell fluid towards a vertical stretching surface," Nonlinear Analysis: Real World Applications, vol. 11, no. 4, pp. 3218-3228, 2010.

[14] M. Khan and A. Shahzad, "On axisymmetric flow of Sisko fluid over a radially stretching sheet," International Journal of Non-Linear Mechanics, vol. 47, pp. 999-1007, 2012.

[15] M. Khan and A. Shahzad, "Falkner-skan boundary layer flow of a sisko fluid," Zeitschrift für Naturforschung A, vol. 67a, pp. 469-478, 2012.

[16] M. Khan, S. Munawar, and S. Abbasbandy, "Steady flow and heat transfer of a Sisko fluid in annular pipe," International Journal of Heat and Mass Transfer, vol. 53, no. 7-8, pp. 1290-1297, 2010.

[17] M. M. Rashidi, T. Hayat, E. Erfani, S. A. M. Pour, and A. A. Hendi, "Simultaneous effects of partial slip and thermal-diffusion and diffusion-thermo on steady MHD convective flow due to a rotating disk," Communications in Nonlinear Science and Numerical Simulation, vol. 16, no. 11, pp. 4303-4317, 2011.

[18] S. Abbasbandy, E. Magyari, and E. Shivanian, "The homotopy analysis method for multiple solutions of nonlinear boundary value problems," Communications in Nonlinear Science and Numerical Simulation, vol. 14, no. 9-10, pp. 3530-3536, 2009. 
[19] T. Hayat and M. Sajid, "Homotopy analysis of MHD boundary layer flow of an upper-convected Maxwell fluid," International Journal of Engineering Science, vol. 45, no. 2-8, pp. 393-401, 2007.

[20] M. Khan, Qurrat-ul-Ain, and M. Sajid, "Heat transfer analysis of the steady flow of an Oldroyd 8constant fluid due to a suddenly moved plate," Communications in Nonlinear Science and Numerical Simulation, vol. 16, pp. 1347-1355, 2011.

[21] M. M. Rashidi, G. Domairry, and S. Dinarvand, "Approximate solutions for the Burger and regularized long wave equations by means of the homotopy analysis method," Communications in Nonlinear Science and Numerical Simulation, vol. 14, no. 3, pp. 708-717, 2009.

[22] M. M. Rashidi, S. A. M. Pour, and S. Abbasbandy, "Analytic approximate solutions for heat transfer of a micropolar fluid through a porous medium with radiation," Communications in Nonlinear Science and Numerical Simulation, vol. 16, no. 4, pp. 1874-1889, 2011.

[23] M. Sajid, M. Awais, S. Nadeem, and T. Hayat, "The influence of slip condition on thin film flow of a fourth grade fluid by the homotopy analysis method," Computers $\mathcal{E}$ Mathematics with Applications, vol. 56, no. 8, pp. 2019-2026, 2008.

[24] Z. Ziabakhsh and G. Domairry, "Analytic solution of natural convection flow of a non-Newtonian fluid between two vertical flat plates using homotopy analysis method," Communications in Nonlinear Science and Numerical Simulation, vol. 14, no. 5, pp. 1868-1880, 2009.

[25] V. Aliakbar, A. Alizadeh-Pahlavan, and K. Sadeghy, "The influence of thermal radiation on MHD flow of Maxwellian fluids above stretching sheets," Communications in Nonlinear Science and Numerical Simulation, vol. 14, pp. 779-794, 2009. 


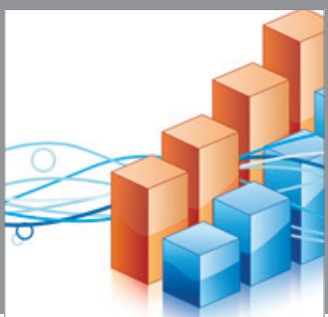

Advances in

Operations Research

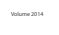

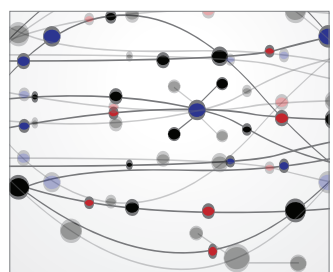

\section{The Scientific} World Journal
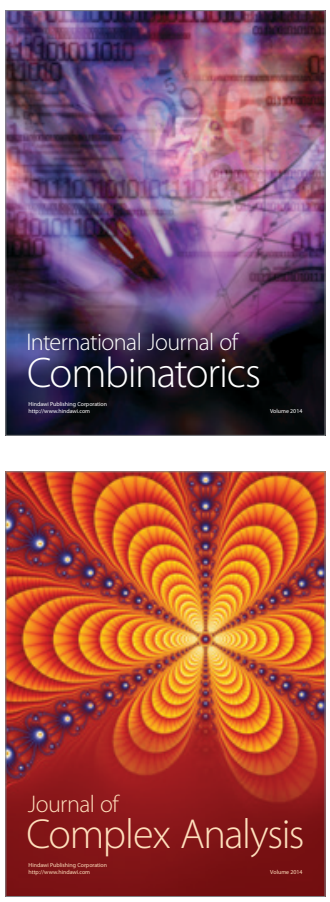

International Journal of

Mathematics and

Mathematical

Sciences
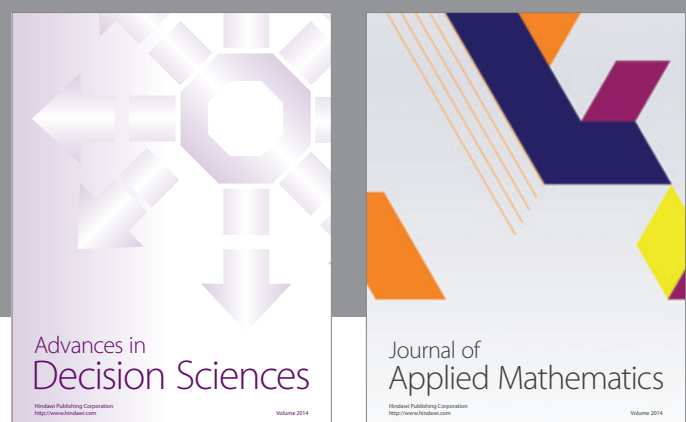

Journal of

Applied Mathematics
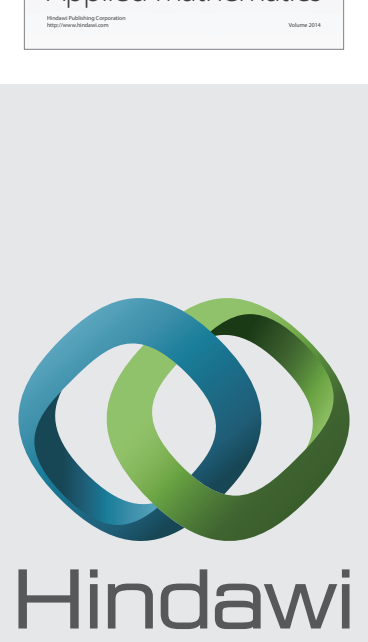

Submit your manuscripts at http://www.hindawi.com
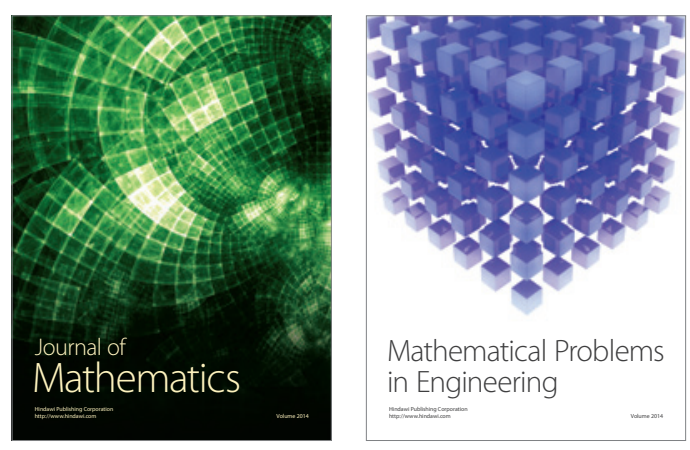

Mathematical Problems in Engineering
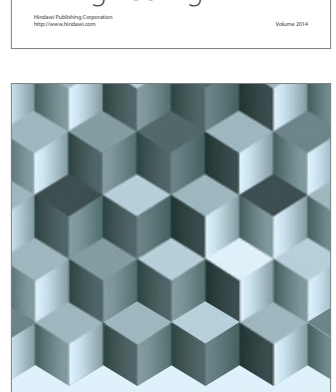

Journal of

Function Spaces
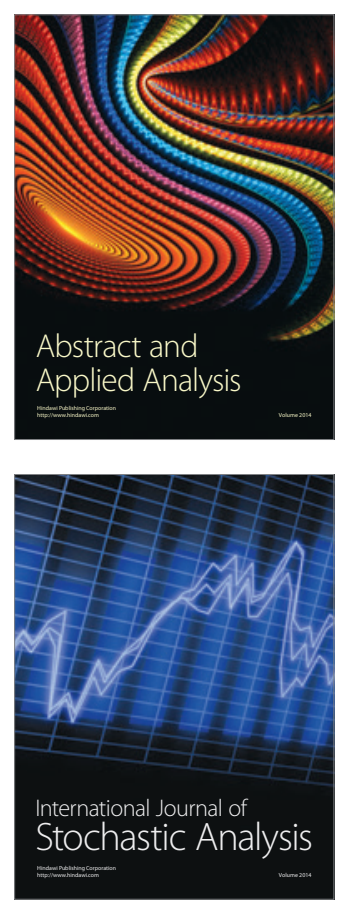

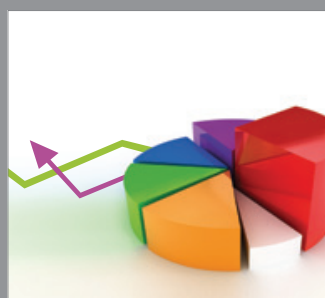

ournal of

Probability and Statistics

Promensencen
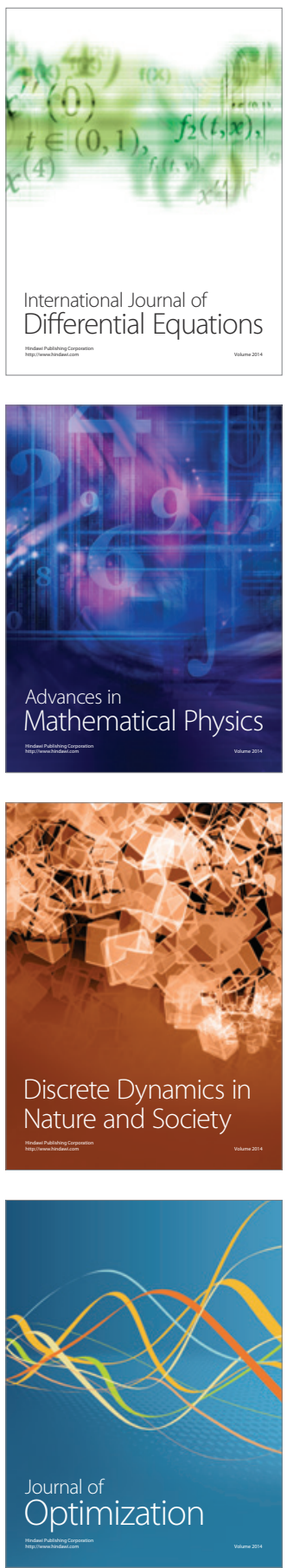